\title{
ON INFINITESIMAL ISOMETRIC DEFORMATIONS
}

\author{
KETI TENENBLAT ${ }^{1}$
}

\begin{abstract}
We consider an analytic $n$-dimensional submanifold $M$ of the Euclidean space $E^{N}$, where $N=n(n+1) / 2$, and we prove the existence of analytic, nontrivial, infinitesimal isometric deformations, in a neighborhood of any point of $M$, which admits a nonasymptotic tangent hyperplane.
\end{abstract}

1. Introduction. Rigidity and isometric deformation problems have been extensively investigated for surfaces in the three dimensional Euclidean space. The theory for submanifolds of the Euclidean space, with codimension one, was developed by E. Cartan [2]. As for submanifolds with higher codimension, very little is known besides the classical rigidity theorem of Allendoerfer [1], which imposes an extremely strong hypothesis on the submanifold. In this paper we consider analytic, $n$-dimensional submanifolds $M$ of the Euclidean space $E^{N}$, where the dimension $N=n(n+1) / 2$ and we investigate the infinitesimal isometric deformation problem.

In \$2, we obtain the system of partial differential equations, which determines an infinitesimal isometric deformation. This is the linearized version of an isometric deformation.

In \$3, we introduce the concept of asymptotic tangent hyperplane at a point of $M$ and we characterize the set of all such hyperplanes.

Finally in $\$ 4$, we prove the following

THEOREM. Let $M$ be an n-dimensional, analytic submanifold of $E^{N}, N=n(n$ $+1) / 2$, with the induced metric and such that the inclusion $i: M \rightarrow E^{N}$ is nondegenerate. If $x \in M$ is such that there exists a nonasymptotic tangent hyperplane at $x$, then there is a neighborhood of $x$ in $M$, which admits an analytic nontrivial infinitesimal isometric deformation.

Other results, involving the notion of asymptotic submanifolds, can be found in [3], [4] and [5].

I am grateful to Professor S. S. Chern for suggesting this problem and my colleague C. E. Harle for helpful comments.

2. Infinitesimal isometric deformations. In this paper, all manifolds and maps are assumed to be analytic. Consider an immersion $f: M \rightarrow E^{N}$, where $M$ is an $n$-dimensional manifold and $E^{N}$ is the Euclidean $N$-dimensional

Received by the editors January 31, 1978.

AMS (MOS) subject classifications (1970). Primary 53B25; Secondary 35A10.

'Partially supported by CNPq. 
space. We define a deformation of the immersion $f$, to be a map $F: I \times M \rightarrow$ $E^{N}$, where $I=[-\varepsilon, \varepsilon]$ for some $\varepsilon>0$, such that for each $t \in I$, the map $F_{t}$ : $M \rightarrow E^{N}$ given by $F_{t}(x)=F(t, x)$ is an immersion and $F_{0}=f$. Each immersion $F_{t}$ induces a Riemannian metric $g_{t}$ on $M$. A deformation $F$ is said to be an isometric deformation if $g_{t}=g_{0}$ for each $t \in I$. Now we consider the linearized version of isometric deformation. A deformation is said to be an infinitesimal isometric deformation if $\left.(d / d t) g_{t}\right|_{t=0}=0$, i.e. for all vector fields $X, Y$ on $M$

$$
\left.\frac{d}{d t}\left\langle F_{t_{*}}(X), F_{t_{*}}(Y)\right\rangle\right|_{t=0}=0
$$

where $\langle$,$\rangle denotes the Euclidean metric.$

Let $F$ be a deformation of an immersion $f: M \rightarrow E^{N}$. For each $x \in M$, let $Z(x)$ be the tangent vector to the curve $t \mapsto F(t, x)$ at $t=0$. We call $Z$ the deformation vector field of $F$. We say that $F$ is a trivial deformation if there exist a skew-symmetric matrix $A$ and a vector $v$ such that for all $x \in M$, $Z(x)=A f(x)+v$.

It is not difficult to see that a deformation $F$ is an infinitesimal isometric deformation if and only if for all tangent vector fields $X, Y$ on $M$

$$
\left\langle D_{X} Z, Y\right\rangle+\left\langle X, D_{Y} Z\right\rangle=0
$$

where $D$ denotes the usual connection in $E^{N}$ (we have identified $M$ with $f(M)$ ). If we denote $Z=T+B$, where $T$ and $B$ are respectively the tangential and normal components of $Z$, then equation (1) is equivalent to

$$
\left\langle\nabla_{X} T, Y\right\rangle+\left\langle\nabla_{Y} T, X\right\rangle+2\langle s(X, Y), B\rangle=0
$$

where $\nabla$ denotes the Riemannian connection on $M$ and $s$ the second fundamental form.

Let $x_{1}, \ldots, x_{n}$ be a coordinate system defined in a neighborhood $V$ of a point $x \in M$. We denote by $\partial / \partial x_{i}$ the coordinate vectors and $g_{i j}=\left\langle\partial / \partial x_{i}\right.$, $\left.\partial / \partial x_{j}\right\rangle, 1<i, j<n$. We choose an orthonormal frame $\xi_{\alpha}$ on $V, \alpha=n+$ $1, \ldots, N$, normal to $M$. We adopt the following indices convention

$$
1<i, j, k<n, \quad n+1<\alpha, \beta, \gamma<N,
$$

and the summation convention with regard to repeated indices. If we consider $T=t^{i} \partial / \partial x_{i}$ and $B=b^{\alpha} \xi_{\alpha}$, then equation (2) is equivalent to the system

$$
\begin{aligned}
g_{i k} \frac{\partial t^{k}}{\partial x_{i}} & +g_{i k} \frac{\partial t^{k}}{\partial x_{j}} \\
& +\left(\left\langle\nabla_{\partial / \partial x_{i}} \frac{\partial}{\partial x_{k}}, \frac{\partial}{\partial x_{j}}\right\rangle+\left\langle\nabla_{\partial / \partial x_{j}} \frac{\partial}{\partial x_{k}}, \frac{\partial}{\partial x_{i}}\right\rangle\right) t^{k}+2 b^{\alpha} h_{i j}^{\alpha}=0
\end{aligned}
$$

where $1<i<j<n$ and $h_{i j}^{\alpha}=\left\langle s\left(\partial / \partial x_{i}, \partial / \partial x_{j}\right), \xi_{\alpha}\right\rangle$.

3. Asymptotic hypersurfaces. Let $M$ be an $n$-dimensional submanifold of $E^{N}, N=n(n+1) / 2$, with the induced metric and such that the inclusion $i$ : $M \rightarrow E^{N}$ is nondegenerate. For a fixed point $x \in M$, an $l$-dimensional, 
$0<l<n$, linear subspace $L$ of the tangent space $T_{x} M$ is called asymptotic if there exists a vector $\xi$ normal to $T_{x} M$, such that for any $X, Y \in L,\langle s(X, Y)$, $\xi\rangle=0$. If $l=n-1$ we have an asymptotic hyperplane at $x$. An $l$-dimensional submanifold $V$ of $M, l<n$, is called asymptotic at $x \in V$ if $T_{x} V$ is asymptotic and asymptotic if this is true for each $x \in V$. The notion of asymptotic submanifolds in a more general context can be found in [3].

The asymptotic hyperplanes at $x \in M$ can be characterized as follows:choose an orthonormal frame $e_{1}, \ldots, e_{N}$ defined on a neighborhood of $x$, such that $e_{1}, \ldots, e_{n}$ are tangent to $M$ and $e_{n+1}, \ldots, e_{N}$ are normal to $M$. Let $\omega^{1}, \ldots, \omega^{N}$ be the dual frame. We denote by $H^{\alpha}$ the second fundamental forms with respect to this frame, i.e. $s(X, Y)=H^{\alpha}(X, Y) e_{\alpha}$. From the theory of a submanifold of an Euclidean space, we know that $\omega^{\alpha}=0$ on $M$ and $H^{\alpha}=h_{i j}^{\alpha} \omega^{i} \otimes \omega^{j}, h_{i j}^{\alpha}=h_{j i}^{\alpha}$. It follows from the definition, that an $(n-1)$-dimensional linear subspace of $T_{x} M$, given by $u_{i} \omega^{i}=0$ is asymptotic if and only if there exists $a_{\alpha} \in \mathbf{R}$, not all zero, such that $a_{\alpha} H^{\alpha}=0$ when restricted to the hyperplane $u_{i} \omega^{i}=0$. It is not difficult to prove that this is equivalent to saying that there exist $a_{\alpha}, c_{i} \in \mathbf{R}$ not all zero, such that

$$
a_{\alpha} H^{\alpha} \equiv u_{i} \omega^{i} \otimes c_{j} \omega^{j}
$$

This reduces to a homogeneous equation in $u_{i}$ of degree $n, P\left(u_{1}, \ldots, u_{n}\right)=0$, which can be described as follows: we consider the matrices

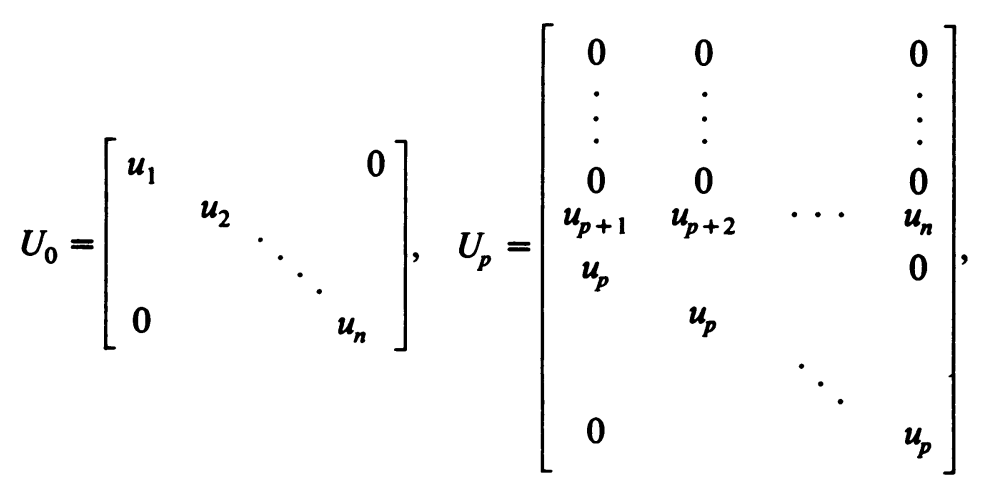

where $1<p<n-1$ and $U_{p}$ has the first $(p-1)$ rows equal to zero.

$$
\begin{aligned}
& A_{0}=\left[\begin{array}{llll}
h_{11}^{n+1} & h_{22}^{n+1} & \cdots & h_{n n}^{n+1} \\
\vdots & \vdots & & \vdots \\
h_{11}^{N} & h_{22}^{N} & \cdots & h_{n n}^{N}
\end{array}\right], \\
& A_{p}=2\left[\begin{array}{cccc}
h_{p p+1}^{n+1} & h_{p p+2}^{n+1} & \cdots & h_{p n}^{n+1} \\
\vdots & \vdots & & \vdots \\
h_{p p+1}^{N} & h_{p p+2}^{N} & \cdots & h_{p n}^{N}
\end{array}\right],
\end{aligned}
$$


$1<p<n-1$. Then

$$
P\left(u_{1}, u_{2}, \ldots, u_{n}\right)=\operatorname{det}\left[\begin{array}{llll}
U_{0} & U_{1} & \cdots & U_{n-1} \\
A_{0} & A_{1} & \ldots & A_{n-1}
\end{array}\right]=0 .
$$

Hence the asymptotic hypersurfaces of $M$ are the solutions of the first order partial differential equation defined by $P\left(u_{1}, \ldots, u_{n}\right)=0$.

In our theorem, we prove that there exist nontrivial, infinitesimal, isometric deformations in a neighborhood of any point of $M$, for which the polynomial $P\left(u_{1}, \ldots, u_{n}\right)=0$ is not an identity.

4. Proof of theorem. The proof is essentially based on the Cauchy-Kowalewsky theorem.

(a) Let $x_{1}, \ldots, x_{n}$ be a coordinate system defined in a neighborhood $V$ of $x$ in $M$, such that the coordinate vectors $\partial / \partial x_{i}$ are orthonormal at $x$, i.e. $g_{i j}(x)=\delta_{i j}$. Moreover we choose an orthonormal frame $\xi_{\alpha}$ in $V$ normal to $M$. We have to prove the local existence of a vector field $Z=t^{i} \partial / \partial x_{i}+b^{\alpha} \xi_{\alpha}$, which satisfies the system of differential equations (3). This system can be written in matrix notation as

$$
\sum_{i=1}^{n} Q^{i} \frac{\partial t}{\partial x_{i}}+R t+2 D b=0
$$

where

$$
t=\left[\begin{array}{c}
t^{1} \\
\vdots \\
t^{n}
\end{array}\right], \quad b=\left[\begin{array}{l}
b^{n+1} \\
\vdots \\
b^{N}
\end{array}\right],
$$

$Q^{i}$ and $R$ are $N \times n$ matrices and $D$ is an $N \times(N-n)$ matrix.

By assumption the inclusion of $M$ in $E^{N}$ is nondegenerate, hence the matrix $D$ is of maximal rank $N-n$. Therefore we can consider (7) as

$$
\sum_{i=1}^{n}\left[\begin{array}{l}
q^{i} \\
\bar{Q}^{i}
\end{array}\right] \frac{\partial t}{\partial x_{i}}+\left[\begin{array}{l}
r \\
\bar{R}
\end{array}\right] t+2\left[\frac{d}{\bar{D}}\right] b=0
$$

where $\bar{D}$ is a nonsingular square matrix in $D$. It follows that

$$
-2=\bar{D}^{-1}\left(\sum_{i=1}^{n} \bar{Q}^{i} \frac{\partial t}{\partial x_{i}}+\bar{R} t\right)
$$

Substituting this relation in the first set of equations in (8) we get

$$
\sum_{i=1}^{n}\left(q^{i}-d \bar{D}^{-1} \bar{Q}^{i}\right) \frac{\partial t}{\partial x_{i}}+\left(r-d \bar{D}^{-1} \bar{R}\right) t=0 .
$$

This is a system of $n$ equations with $n$ unknowns to which we apply the Cauchy-Kowalewsky theorem. 
The characteristic equation for (10) is given by

$$
\operatorname{det}\left[\sum_{i=1}^{n}\left(q^{i}-d \bar{D}^{-1} \bar{Q}^{i}\right) u_{i}\right]=0
$$

In (b) we prove that at the point $x$ this equation is equivalent to $P\left(u_{1}, \ldots, u_{n}\right)=0$ given in (6). Therefore the characteristic hypersurfaces of the system (10) are the asymptotic hypersurfaces of the manifold $M$.

We consider a hypersurface $S$ of $M$, which is not asymptotic and contains $x$ (exists by assumption). We observe that if a deformation vector field $Z$ is trivial, then the tangential component of $Z$ is of the form

$$
\sum_{j, k}\left\langle A i+v, \frac{\partial}{\partial x_{k}}\right\rangle g^{k j} \frac{\partial}{\partial x_{j}}
$$

where $i: M \rightarrow E^{N}$ is the inclusion map, $A$ is a fixed skew-symmetric matrix, $v$ is a vector in $E^{N}$ and $g^{k j}$ is the inverse matrix of $g_{k j}$. Since the space of vector fields of the form (12), along the submanifold $S$ is finite dimensional, we can always choose an analytic tangential vector field $t^{j} \partial / \partial x_{j}$ in a neighborhood of $x$ along $S$ which is not of the form (12). With this initial condition fixed, it follows from the Cauchy-Kowalewsky theorem, that locally there exists a unique analytic tangential vector field satisfying (10) and the initial condition. Next using (9) we determine the normal component and hence we get a nontrivial deformation vector field $Z$. Therefore for $\varepsilon>0$ sufficiently small and $\bar{V}$ a neighborhood of $x$ in $M$, the map $F(r, y)=i(y)+r Z(y), r \in$ $(-\varepsilon, \varepsilon)$ and $y \in \bar{V}$ is a nontrivial infinitesimal isometric deformation of the inclusion $i: M \rightarrow E^{N}$.

(b) It remains to prove that at $x$ (11) is equivalent to (6). To fix ideas we assume the following order for the indices $i, j$ of the system of equations (3):

$$
\begin{aligned}
& i=j=1, \ldots, n ; \quad i=1, j=2, \ldots, n ; \\
& i=2, j=3, \ldots, n, \ldots, i=n-1, j=n .
\end{aligned}
$$

With this order, we can describe the matrices $D$ and $Q^{i}$ of equation (7) as

$$
2 D=\left[\begin{array}{cc}
2 & { }^{t} A_{0} \\
& { }^{t} A_{1} \\
& \vdots \\
& { }^{t} A_{n-1}
\end{array}\right], \quad Q^{i}=\left[\begin{array}{c}
G_{0}^{i} \\
G_{1}^{i} \\
\vdots \\
G_{n-1}^{i}
\end{array}\right]
$$

where ${ }^{t} A_{0}, \ldots,{ }^{t} A_{n-1}$ are the transpose matrices of (5); $G_{0}^{i}$ is an $n \times n$ matrix defined by 


$$
G_{0}^{i}=\left[\begin{array}{lllll}
0 & 0 & \ldots & 0 & \\
\vdots & \vdots & & \vdots & \\
0 & 0 & & 0 & \\
2 g_{1 i} & 2 g_{2 i} & \cdots & 2 g_{n i} & \\
0 & 0 & & & 0 \\
\vdots & \vdots & & \vdots & \\
0 & 0 & \cdots & 0 &
\end{array}\right]
$$

where the nonzero elements are on the $i$ th row; and $G_{p}^{i}, 1<p<n-1$, is an $(n-p) \times n$ matrix defined by

$$
\begin{aligned}
& \text { if } p<i \quad G_{p}^{i}=\left[\begin{array}{cccc}
0 & 0 & & 0 \\
\vdots & \vdots & & \vdots \\
0 & 0 & & 0 \\
g_{1 p} & g_{2 p} & \cdots & g_{n p} \\
0 & 0 & & 0 \\
\vdots & \vdots & & \vdots \\
0 & 0 & \cdots & 0
\end{array}\right] \leadsto(i-p) \text { th row, } \\
& \text { if } p=i \quad G_{p}^{i}=\left[\begin{array}{lll}
g_{1 i+1} & \cdots & g_{n i+1} \\
g_{1 i+2} & \cdots & g_{n i+2} \\
\vdots & & \vdots \\
g_{1 n} & \cdots & g_{n n}
\end{array}\right] \text {, } \\
& \text { if } p>i \quad G_{p}^{i}=0 \text {. }
\end{aligned}
$$

With the same notation as in part (a) we consider the product of the matrices

$$
\left[\begin{array}{cc}
I_{n} & -d \bar{D}^{-1} \\
0 & \bar{D}^{-1}
\end{array}\right]\left[\begin{array}{cc}
\Sigma_{i} q^{i} u_{i} & 2 d \\
\Sigma_{i} \bar{Q}^{i} u_{i} & 2 \bar{D}
\end{array}\right]=\left[\begin{array}{cc}
\Sigma_{i}\left(q^{i}-d \bar{D}^{-1} \bar{Q}^{i}\right) u_{i} & 0 \\
* & 2 I_{N-n}
\end{array}\right]
$$

where $I_{n}$ denotes the $n \times n$ identity matrix. Therefore $u_{i}$ satisfy (11) if and only if

$$
\operatorname{det}\left[\sum_{i} Q^{i} u_{i} 2 D\right]=0
$$

Now we remark that the coordinate system was chosen, such that at $x$, $g_{i j}(x)=\delta_{i j}$ and it can easily be verified that 


$$
\sum_{i} Q^{i}(x) u_{i}=\left[\begin{array}{c}
2^{t} U_{0} \\
{ }^{t} U_{1} \\
\vdots \\
U_{n-1}
\end{array}\right]
$$

where ${ }^{t} U_{0} \ldots{ }^{\prime} U_{n-1}$ are the transpose matrices of (4). Therefore it follows from (13), that at $x(11)$ is equivalent to (6). Q.E.D.

\section{REFERENCES}

1. C. B. Allendoerfer, Rigidity for spaces of class greater than one, Amer. J. Math. 61 (1939), 633-644.

2. E. Cartan, La déformation des hypersurfaces dans l'espace euclidien reél à $n$ dimensions, Bull. Soc. Math. France 44 (1916), 65-99.

3. H. Jacobowitz, Deformations leaving a hypersurface fixed, Proc. Sympos. Pure Math., vol. 23, Amer. Math. Soc., Providence, R. I., 1971, pp. 343-351.

4. K. Tenenblat, A rigidity theorem for three dimensional submanifolds in Euclidean six space, J. Differential Geometry 74 (1978), 507-517.

5. __, On characteristic hypersurfaces of submanifolds in Euclidean space, Pacific J. Math. (to appear).

Departagnt of Mathematics, University of California, Berkoley, Californi 94720

Departamento de Matźmaticas I.E., Universidade de Brasila, 70910 Brasiuh, D. F. BrAsII (Current address) 